\title{
Energy level shifts in two-step spin-orbit coupling ab initio calculations
}

\author{
Goar Sánchez-Sanz \\ Departamento de Química, Universidad Autónoma de Madrid, 28049 Madrid, Spain \\ Zoila Barandiarán and Luis Seijo* \\ Departamento de Química, Universidad Autónoma de Madrid, 28049 Madrid, Spain and \\ Instituto Universitario de Ciencia de Materiales Nicolás Cabrera, \\ Universidad Autónoma de Madrid, 28049 Madrid, Spain
}

(Dated: August 2, 2010)

\begin{abstract}
We point out a problem with two-step spin-orbit ab initio calculations in which the energy levels of spin-orbit free Hamiltonians are shifted as a means to including dynamic correlations at low cost in small spin-orbit configuration interaction calculations. The usual shifts driven by the energy order of the states can lead to anomalous results when avoided crossings exist with significant change of wave function character, which take place at different nuclear positions in the configurational spaces of the first and the second steps. In these cases, the shifts of the spin-orbit free energy levels must be assigned according to the characters of the wave functions.
\end{abstract}

PACS numbers: 31.15.A-, 31.15.aj, 31.15.am

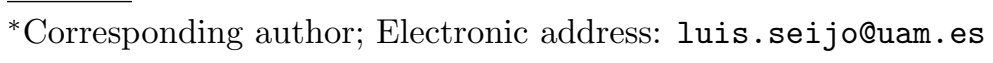




\section{INTRODUCTION}

Two-step spin-orbit coupling ab initio methods have been proposed -and are presently under use- as a means of achieving a reasonable balance between accuracy and economy in electronic structure calculations in which both dynamic correlation and spin-orbit coupling are important effects. ${ }^{1-3}$ These methods appeared as an answer to the problem associated with the fact that spin symmetry breaking leads to much larger configuration interaction (CI) matrices to handle with spin-orbit Hamiltonians than with spin-free Hamiltonians. They are based on two ideas: first, that dynamic electron correlation and spin-orbit coupling are largely uncoupled in a vast majority of atomic, molecular, and solid state systems, and, second, that these two effects can be considered in two different steps of the electronic structure calculation because they pose very different demands in terms of electronic configuration space. This has been recognized and implemented in early two-step perturbation theory/CI methods, ${ }^{4}$ in which a first step consisting of a conventional correlated calculation with a spin-free Hamiltonian was followed by a second step, where the spin-orbit Hamiltonian was used and the correlation effects from the first calculation were conveniently transferred under some formal conditions of the wave functions, like contracted CI.

Later, on the basis of the above ideas, Llusar et al. ${ }^{1}$ formulated a simple, general, effective Hamiltonian to be used in the second step, in spin-orbit (relatively small) CI calculations with all types of many-electron basis sets, either contracted or uncontracted CI, determinantal, double group adapted configuration space, etc. This effective Hamiltonian is simply made of the original Hamiltonian plus an operator that shifts the eigenstates of the spin-free part of the Hamiltonian, within any configuration space, to their original energies. This was called spin-free-state shifting operator. Vallet et al. ${ }^{2}$ and Malmqvist et al. ${ }^{3}$ adopted the use of such a shifting operator in a two-step uncontracted determinantal effective Hamiltonian spin-orbit CI method (implemented in EPCISO) ${ }^{2}$ and a two-step restricted-active-space state-interaction spin-orbit method, RASSI-SO ${ }^{3}$ (implemented in MOLCAS). ${ }^{5}$

In this letter, we point out that the usual calculations of the shifting constants in the spinfree-state shifting operator, that is, the energy shifts of the spin-orbit free levels, which are driven by their energy order within each irreducible representation, can lead to anomalous results when avoided crossings exist with significant change of character of the wave functions at each side. In these cases, the shifts of the spin-orbit free energy levels must be assigned 
according to the character of the wave functions rather than according to their energy order.

\section{METHOD AND RESULTS}

Basically, in two-step spin-orbit methods an effective Hamiltonian is used which is made of three operators: the spin-free Hamiltonian $\hat{H}^{S F}$, the spin-orbit coupling Hamiltonian $\hat{H}^{S O}$, and the energy shift operator $\hat{H}^{\text {shift }}$,

$$
\hat{H}_{e f f}=\hat{H}^{S F}+\hat{H}^{S O}+\hat{H}^{\text {shift }} .
$$

$\hat{H}^{S F}+\hat{H}^{S O}$ is the regular Hamiltonian of the electronic system, $\hat{H}$, and $\hat{H}^{\text {shift }}$ is defined as

$$
\hat{H}^{\text {shift }}=\sum_{i S M_{S} \Gamma \gamma} \delta_{i S \Gamma}\left|\Phi_{i S M_{S} \Gamma \gamma}^{\mathcal{P}}\right\rangle\left\langle\Phi_{i S M_{S} \Gamma \gamma}^{\mathcal{P}}\right|
$$

with

$$
\delta_{i S \Gamma}=\left[E_{i S \Gamma}^{\mathcal{G}}-E_{G S}^{\mathcal{G}}\right]-\left[E_{i S \Gamma}^{\mathcal{P}}-E_{G S}^{\mathcal{P}}\right]
$$

In equations 2 and $3, S$ and $M_{S}$ are spin quantum numbers and $\Gamma$ and $\gamma$ are an irreducible representation of the system's symmetry group and one of its subspecies. $i$ is an ordinal number used to label the different energy levels with the same values of $S M_{S} \Gamma \gamma$. The values of $i$ are normally chosen following the energy order of the levels. $\mathcal{G}$ stands for a configurational space of large size and $\mathcal{P}$ stands for a configurational space of smaller size. The space $\mathcal{G}$ is required to give the right correlation effects for the energy spectrum of $\hat{H}$ and the space $\mathcal{P}$ is required to give the right spin-orbit couplings between the eigenfunctions of the spin-free Hamiltonian $\hat{H}^{S F}$. This definition of $\hat{H}^{\text {shift }}$ is such that the spin-orbit free effective Hamiltonian $\hat{H}^{S F}+\hat{H}^{\text {shift }}$ has, in the small configurational space $\mathcal{P}$, the same energy spectrum as $\hat{H}^{S F}$ has in the large configurational space $\mathcal{G}$, as shown in Fig. 1. This is the right spin-orbit free energy spectrum for the calculation of spin-orbit splittings. The other basic ingredients for the calculation of these splittings are the spin-orbit couplings. The choice of the configurational space $\mathcal{P}$ makes the wave functions $\Phi_{i S M_{S} \Gamma \gamma}^{\mathcal{P}}$ appropriate for the couplings. Now, since these wave functions are eigenfunctions of $\hat{H}^{S F}+\hat{H}^{\text {shift }}$ in $\mathcal{P}, \hat{H}^{S F}+\hat{H}^{\text {shift }}$ is the right spin-orbit free effective Hamiltonian to which the spin-orbit coupling Hamiltonian must be added in order to get a full effective Hamiltonian $\hat{H}_{e f f}$ that will be represented in the small configurational space $\mathcal{P}$. 
At this point, we must recall that the previous arguments stand as long as the wave functions of the large space $\mathcal{G}$ and of the small space $\mathcal{P}$ have the correct parentage, which means that an approximate spin-orbit coupling $\left\langle\Phi_{i S M_{S} \Gamma \gamma}^{\mathcal{P}}\left|\hat{H}^{S O}\right| \Phi_{j S^{\prime} M_{S}^{\prime} \Gamma^{\prime} \gamma^{\prime}}^{\mathcal{P}}\right\rangle$ goes together with the right energy difference $E_{i S \Gamma}^{\mathcal{G}}-E_{j S^{\prime} \Gamma^{\prime}}^{\mathcal{G}}$, because this is the energy difference that would accompany the spin-orbit coupling $\left\langle\Phi_{i S M_{S} \Gamma \gamma}^{\mathcal{G}}\left|\hat{H}^{S O}\right| \Phi_{j S^{\prime} M_{S}^{\prime} \Gamma^{\prime} \gamma^{\prime}}^{\mathcal{G}}\right\rangle$ in the one-step spin-orbit calculation in the large space $\mathcal{G}$ that the two-step calculation intends to mimic.

However, the choice of the ordinal number $i$ following the energy order of the levels with the same $S M_{S} \Gamma \gamma$ does not guarantee the correct parentage we have just mentioned. This is the case when states of the same symmetry but with different electronic nature have avoided crossings and the location of the crossings in the $\mathcal{G}$ and $\mathcal{P}$ spaces do not coincide. In these cases, in the nuclear configurations between the avoided crossings in the $\mathcal{G}$ and $\mathcal{P}$ spaces, wave functions of different electronic nature in the two spaces are linked if the energy order of the levels is used as the correspondence criterion, which results in wrong spin-orbit couplings and, in consequence, in wrong spin-orbit splittings. In order to solve these problems, a different correspondence criterion must be used which takes into account the electronic nature of the $\mathcal{G}$ and $\mathcal{P}$ wave functions. Ideally, $\Phi_{i S M_{S} \Gamma \gamma}^{\mathcal{P}}$ should resemble $\Phi_{i S M_{S} \Gamma \gamma}^{\mathcal{G}}$ as much as possible. A maximum overlap criterion should be the simplest one for this purpose; according to it, $\Phi_{i S M_{S} \Gamma \gamma}^{\mathcal{P}}$ is not chosen as the wave function in space $\mathcal{P}$ that has the $i$-th energy in the $S M_{S} \Gamma \gamma$ symmetry block, but as the one that has maximum overlap with $\Phi_{i S M_{S} \Gamma \gamma}^{\mathcal{G}}$. In many cases, inspection of the configurational character of the wave functions is sufficient to solve these assignments.

The comments in the previous paragraph are illustrated in Fig. 2. In this figure, we show energy levels of the $\left(\mathrm{YbCl}_{8}\right)^{6-}$ cluster embedded in the solid host $\mathrm{SrCl}_{2}$ as a function of the $\mathrm{Yb}-\mathrm{Cl}$ distance. ${ }^{6}$ These levels are a key to understanding the luminescence properties of the phosphor material Yb-doped $\mathrm{SrCl}_{2} \cdot{ }^{7}$ Panels (a) and (b) correspond to spin-orbit free results of the first step calculations, in particular to the ${ }^{1} T_{1 u}$ symmetry block of the group $O_{h}$, which is specially relevant because the ${ }^{1} A_{1 g} \rightarrow^{1} T_{1 u}$ are the only electric dipole allowed absorptions (the ground state is ${ }^{1} A_{1 g}$ ). (a) shows the small space $\mathcal{P}$ results and (b) shows the large space $\mathcal{G}$ results. [The $\mathcal{G}$ calculation is a state-average complete-activespace self-consitent-field ${ }^{8}$ multi-state second-order perturbation theory ${ }^{9,10}$ calculation, SACASSCF/MS-CASPT2, with a CAS space made of 14 electrons in the 13 molecular orbitals with main character of $\mathrm{Yb}-4 f, \mathrm{Yb}-5 d$, and $\mathrm{Yb}-6 s$ and all valence electrons correlated. The 
$\mathcal{P}$ calculation is a $\mathrm{MRCI}(\mathrm{S})$ calculation with a multireference made of the restricted-activespace $4 f^{13} 5 d^{1}$ and $4 f^{13} 6 s^{1}$.] It is clear that the $\mathcal{P}$ calculation in (a) has an avoided crossing between the levels $5{ }^{1} T_{1 u}$ and $6{ }^{1} T_{1 u}$ at around $3.12 \AA$, whereas the same avoided crossing appears beyond $3.20 \AA$ in the $\mathcal{G}$ calculation in (b). In the $\mathcal{G}$ results, up to $3.20 \AA, 5{ }^{1} T_{1 u}$ has a dominant character of the configuration $4 f^{13} 5 d\left(t_{2 g}\right)^{1}$, whereas $6{ }^{1} T_{1 u}$ has a dominant $4 f^{13} 6 s^{1}$ character $\left[4 f, 5 d\left(t_{2 g}\right)\right.$, and $6 s$ stand for $\left(\mathrm{YbCl}_{8}\right)^{6-}$ molecular orbitals with main character $\mathrm{Yb}-4 f, \mathrm{Yb}-5 d$ of $t_{2 g}$ symmetry, and $\mathrm{Yb}-6 s$, respectively.] The two different electronic natures lead to two very different equilibrium distances and to the avoided crossings. In the $\mathcal{P}$ results, the configurational characters of $5^{1} T_{1 u}$ and $6{ }^{1} T_{1 u}$ are the same than in the $\mathcal{G}$ results up to a bond distance of $3.12 \AA$, but the characters flip above this value: $5^{1} T_{1 u}$ becomes $4 f^{13} 6 s^{1}$ and $6{ }^{1} T_{1 u}$ becomes $4 f^{13} 5 d\left(t_{2 g}\right)^{1}$.

The spin-orbit calculations of the second step with the effective Hamiltonian (Eq. 1), using conventional energy shifts driven by the energy order, are shown in panel (c), where the anomalous effects of the mismatches between $\mathcal{G}$ and $\mathcal{P}$ wave functions and energies at distances longer than $3.12 \AA$ are evident in the shape of the level $21 T_{1 u}$ of the $O_{h}^{\prime}$ double group, which is a potential fast light emitter. Although less evident and important from the physical point of view in this system, the anomalies are also visible in levels $14 T_{1 u}$ and $18 T_{1 u}$.

Using the new correspondence criterion in this case implies that, above $3.12 \AA$, the small space $\mathcal{P}$ wave functions $\Phi_{5^{1} T_{1 u} M_{S} \gamma}^{\mathcal{P}}$ and $\Phi_{6^{1} T_{1 u} M_{S} \gamma}^{\mathcal{P}}$ to be used in

$$
\delta_{5^{1} T_{1 u}}\left|\Phi_{5^{1} T_{1 u} M_{S} \gamma}^{\mathcal{P}}\right\rangle\left\langle\Phi_{5^{1} T_{1 u} M_{S} \gamma}^{\mathcal{P}}\left|+\delta_{6^{1} T_{1 u}}\right| \Phi_{6^{1} T_{1 u} M_{S} \gamma}^{\mathcal{P}}\right\rangle\left\langle\Phi_{6^{1} T_{1 u} M_{S} \gamma}^{\mathcal{P}}\right|
$$

will be, respectively, the sixth and the fifth ${ }^{1} T_{1 u} M_{S} \gamma \mathcal{P}$ eigenfunctions (instead of the fifth and the sixth,) while the values of the $\delta_{5^{1} T_{1 u}}$ and $\delta_{6^{1} T_{1 u}}$ parameters are the same as before. Doing this corrects the anomalies, as shown in panel (d) of Fig. 2. Now, the equilibrium structure and breathing vibrational frequency of the $21 T_{1 u}$ can be safely computed; these are key ingredients for the simulation of the fast emission from this potential metastable state to the ground state. 


\section{CONCLUSIONS}

We have shown that two-step spin-orbit coupling ab initio calculations with energy shifts driven by energy order may lead to anomalous results when avoided crossings exist that appear at different nuclear configurations in the large space and the small space calculations. The problems are solved when the shifts of the energy levels are assigned according to the characters of the wave functions rather than according to their energy order. Then, reprogramming correspondences between large and small space energy levels in calculations of this type is required in order to guarantee correct results in mass production calculations. Alternatively, new two-step spin-orbit coupling methods with intrinsically correct correspondences between large and small space energy levels may be developed.

\section{Acknowledgments}

This work was partly supported by a grant from Ministerio de Ciencia e Innovación, Spain (Dirección General de Programas y Transferencia de Conocimiento MAT2008-05379/MAT). G.S.-S. acknowledges a FPI fellowship from Ministerio de Ciencia e Innovación, Spain.

1 R. Llusar, M. Casarrubios, Z. Barandiarán, and L. Seijo, J. Chem. Phys. 105, 5321 (1996).

2 V. Vallet, L. Maron, C. Teichteil, and J.-P. Flament, J. Chem. Phys. 113, 1391 (2000).

3 P. A. Malmqvist, B. O. Roos, and B. Schimmelpfennig, Chem. Phys. Lett. 357, 230 (2002).

4 C. Teichteil, M. Pelissier, and F. Spiegelmann, Chem. Phys. 81, 273 (1983).

5 G. Karlström, R. Lindh, P. A. Malmqvist, B. O. Roos, U. Ryde, V. Veryazov, P. O. Widmark, M. Cossi, B. Schimmelpfennig, P. Neogrady, and L. Seijo, Comput. Mater. Sci. 28, 22 (2003).

6 G. Sánchez-Sanz, L. Seijo, and Z. Barandiarán, in preparation.

7 T. S. Piper, J. P. Brown, and D. S. McClure, J. Chem. Phys. 46, 1353 (1967).

8 B. O. Roos, P. R. Taylor, and P. E. M. Siegbahn, Chem. Phys. 48, 157 (1980); P. E. M. Siegbahn, A. Heiberg, J. Almlöf, and B. O. Roos, J. Chem. Phys. 74, 2384 (1981); P. Siegbahn, A. Heiberg, B. Roos, and B. Levy, Phys. Scr. 21, 323 (1980).

9 A. Zaitsevskii and J. P. Malrieu, Chem. Phys. Lett. 233, 597 (1995). 
10 J. Finley, P.-Å. Malmqvist, B. O. Roos and L. Serrano-Andrés, Chem. Phys. Lett. 288, 299 (1998). 
FIG. 1: Schematic representation of the energy levels and shifts within a given symmetry block $S M_{S} \Gamma \gamma$.

FIG. 2: Energy levels of the $\left(\mathrm{YbCl}_{8}\right)^{6-}$ cluster embedded in $\mathrm{SrCl}_{2}$ as a function of the $\mathrm{Yb}-\mathrm{Cl}$ distance. (a) ${ }^{1} T_{1 u}$ levels in spin-orbit free CI calculations (small space $\mathcal{P}$ results). (b) ${ }^{1} T_{1 u}$ levels in spin-orbit free CASPT2 calculations (large space $\mathcal{G}$ results). In (a) and (b), full lines are used for wave functions with $4 f^{13} 5 d\left(t_{2 g}\right)^{1}$ main character and dashed lines for wave functions with $4 f^{13} 6 s^{1}$ main character. (c) and (d) $T_{1 u}$ levels in spin-free-state-shifted spin-orbit CI calculations; (c) corresponds to the usual shifts, driven by energy order within each spin and spatial irreducible representation; (d) corresponds to the new shifts, driven by the character of the spin-orbit free wave functions. 


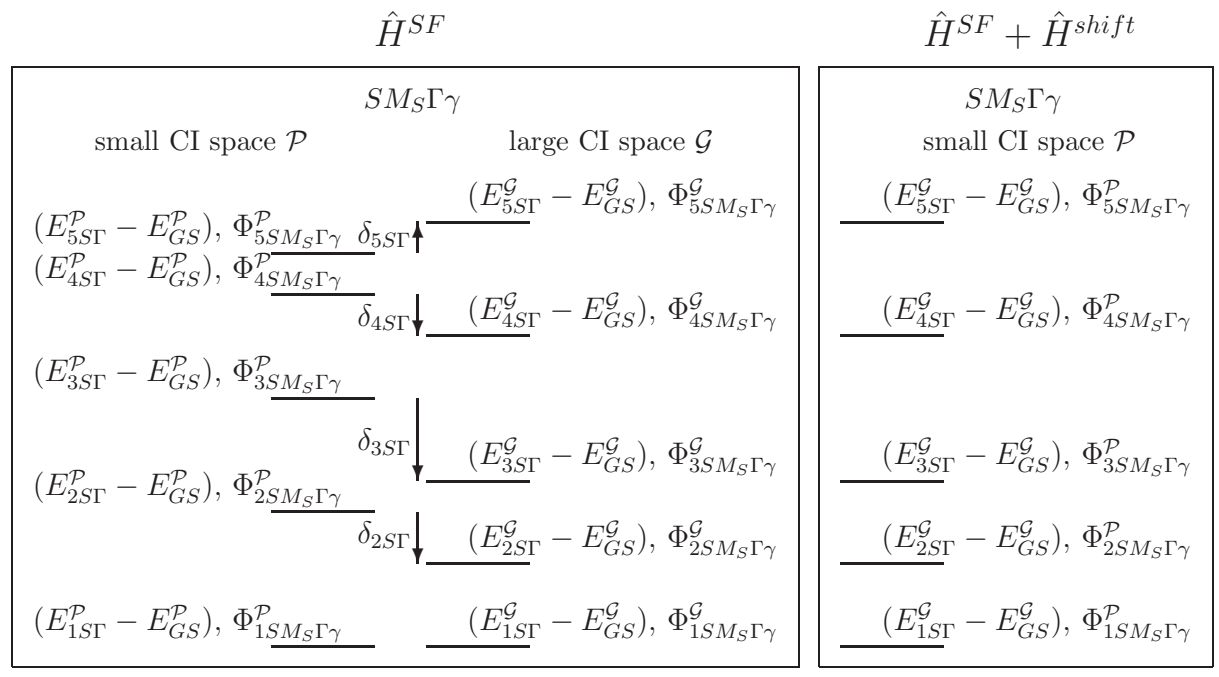

Figure 1 Sánchez-Sanz et al. 

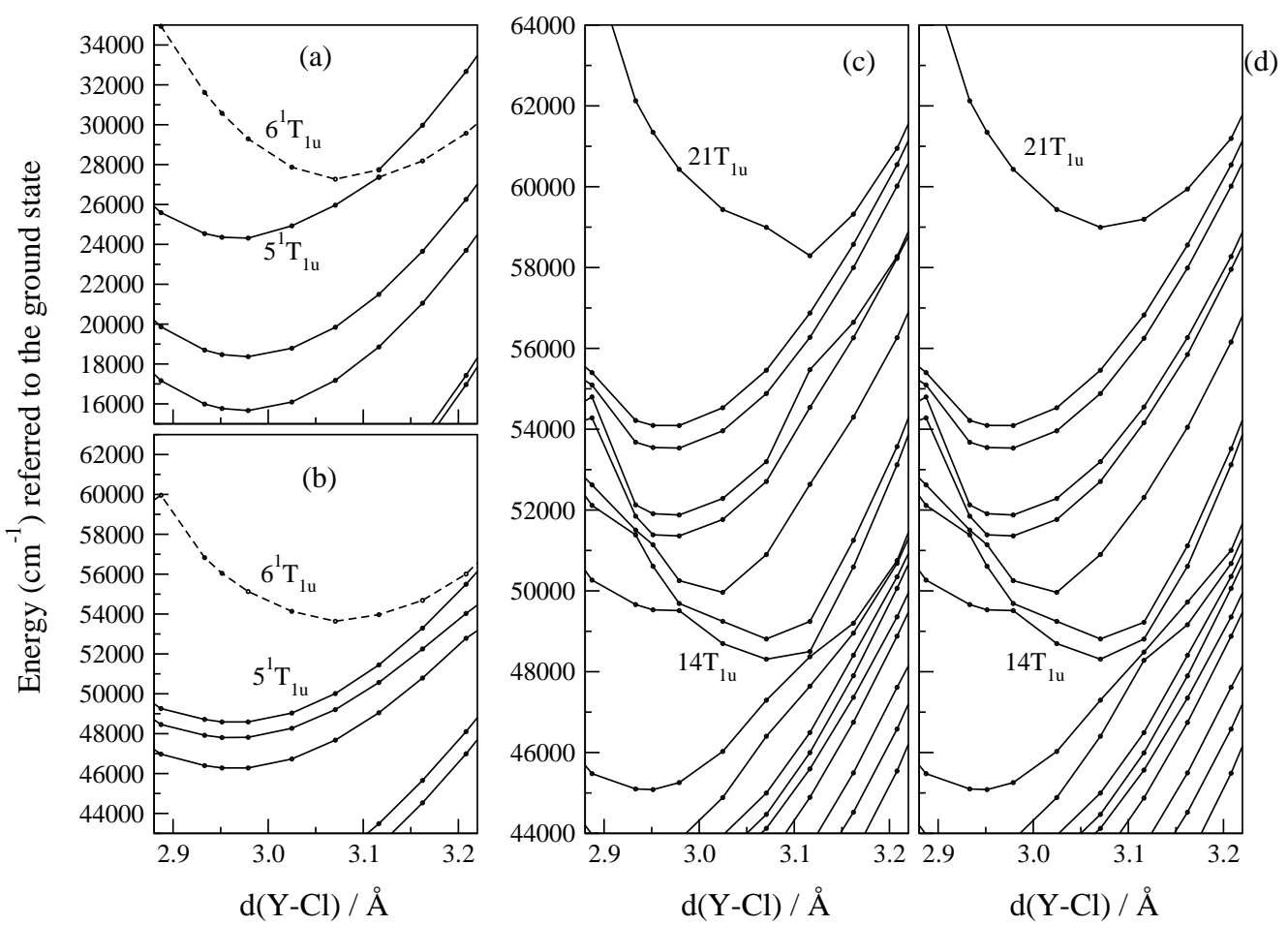

Figure 2 Sánchez-Sanz et al. 